\title{
QUASI-TWO-DIMENSIONAL APPROXIMATION FOR NUMERICAL SIMULATION OF FLOWS IN ENGINE DUCTS
}

\section{Vlasenko and A. Shiryaeva}

Central Aerohydrodynamic Institute 1 Zhukovsky Str., Zhukovsky, Moscow Region 140180, Russia

\begin{abstract}
New quasi-two-dimensional (2.5D) approach to description of threedimensional (3D) flows in ducts is proposed. It generalizes quasi-onedimensional (quasi-1D, 1.5D) theories. Calculations are performed in the $(x ; y)$ plane, but variable width of duct in the $z$ direction is taken into account. Derivation of $2.5 \mathrm{D}$ approximation equations is given. Tests for verification of $2.5 \mathrm{D}$ calculations are proposed. Parametrical $2.5 \mathrm{D}$ calculations of flow with hydrogen combustion in an elliptical combustor of a high-speed aircraft, investigated within HEXAFLY-INT international project, are described. Optimal scheme of fuel injection is found and explained. For one regime, 2.5D and 3D calculations are compared. The new approach is recommended for use during preliminary design of combustion chambers.
\end{abstract}

\section{INTRODUCTION}

Turbulent flows of viscous gas in ducts (especially, in aircraft power plants) are, as a rule, essentially 3D, complex, multiscale phenomena that can contain boundary layers, recirculation zones, mixing layers and jets, compression and rarefaction waves, finite-rate reactions and unsteady processes. Calculation of such flows on the basis of full 3D Reynolds-averaged Navier-Stokes (RANS) equations requires huge computer resources even in the case of parallel computing. As a result, multiple parametrical calculations, which are necessary on the stage of combustor design, are impossible. Even now, simple engineering estimations, based on empirical relations, and calculations in quasi-1D approximation are often used for prediction of combustor characteristics for the design purposes. However, quasi-1D approximation cannot take into account some specific features resulting from non-1D character of flow, e. g., nonuniformity of heat

(C) The Authors, published by EDP Sciences. This is an open access article distributed under the terms of the Creative Commons Attribution License 4.0 (http://creativecommons.org/licenses/by/4.0/). 
release depending on mixing of fuel with air flow and on the heat turbulent transport across the duct. To improve accuracy and informativeness of approximate parametrical calculation on the stage of design, approximation of $2.5 \mathrm{D}$ flow may be used.

The term "2.5D approximation" means that for the description of 3D processes, the $2 \mathrm{D}$ equations, taking into account flow nonuniformity in the third spatial direction, are used. In this sense, classical equation system for axisymmetric flows is also 2.5D approach for the description of 3D flow. But it is applicable only if the problem geometry is axisymmetric and if assumption about the absence of flow swirl is acceptable. Literature review has allowed to find other examples of 2.5D approach to description of 3D flows. Probably, the best known is the "method of flat sections" for the description of flow around infinite swept wing (see, for example, [1-4]). The 2.5D approach is used in aeroacoustics for the description of $3 \mathrm{D}$ sonic wave propagation, when the basic aerodynamic flow has two-dimensional (2D) character $[5,6]$. There are techniques, analogous to method of flat sections, for description of stratified flows with given character of flow variation from one layer to another $[7,8]$. But all these approaches cannot be used for the description of flows in ducts.

In this work, new $2.5 \mathrm{D}$ approach to the description of $3 \mathrm{D}$ flows in ducts is proposed. This approach generalizes quasi-1D ("1.5D") theories. In $2.5 \mathrm{D}$ approximation, the real $3 \mathrm{D}$ flow is replaced by a flow where all parameters are constant along $z$ axis. It may be treated as a result of $3 \mathrm{D}$ flow averaging along $z$. Calculations are performed in the $(x ; y)$ plane but variable width of duct in $z$ direction is taken into account.

\section{QUASI-TWO-DIMENSIONAL FLOW EQUATION SYSTEM}

The flow in a duct of arbitrary geometry is considered (Fig. 1). The duct geometry is projected on some plane. In this plane, Cartesian coordinate system $(x ; y)$ is introduced. Spatial direction of the third coordinate axis, $z$, is determined using the right-hand screw rule. Computational domain is projection of the duct geometry on the $(x ; y)$ plane. It is covered by computational grid (see Fig. 1). Spatial curve on the duct surface, corresponding to the contour of the duct projection on the $(x ; y)$ plane, subdivides the duct surface into two halves. These halves can be described by functions $z^{+}(x, y)$ ("front" surface) and $z^{-}(x, y)$ ("back" surface). For each pair of values $(x ; y), z^{+} \geq z^{-}$. If the duct is symmetrical about vertical plane, $z^{+} \geq 0$ and $z^{-}=-z^{+}$.

Reynolds-averaged Navier-Stokes equation system for multicomponent gas with finite-rate reactions, closed by differential model of turbulence and by model 


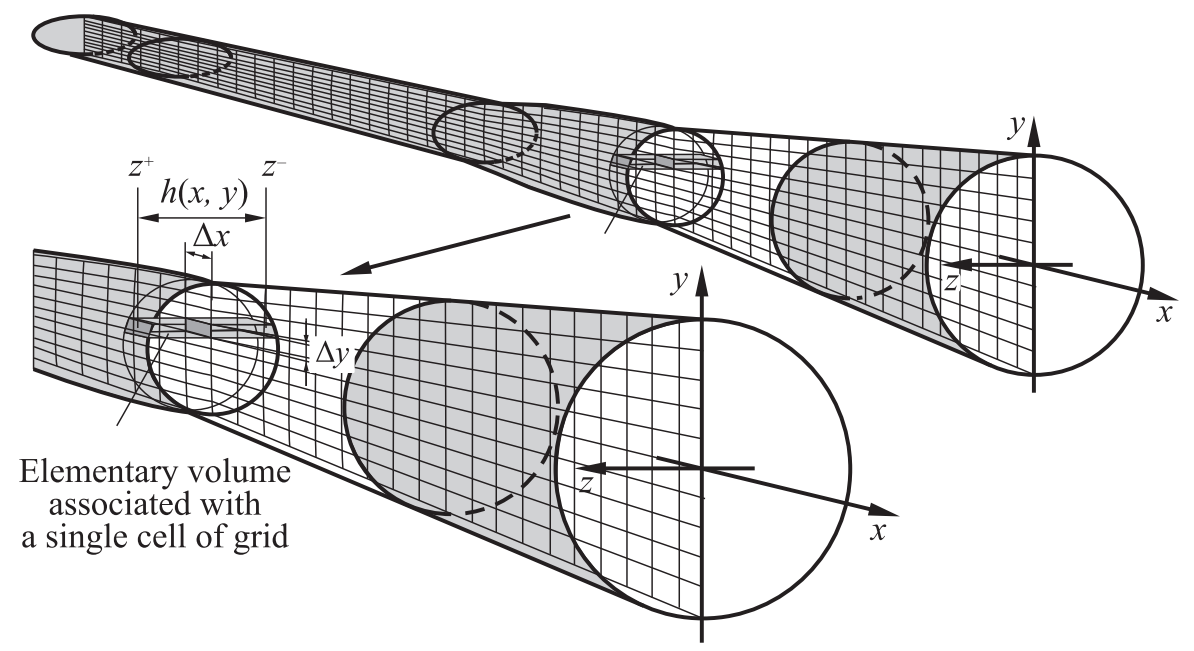

Figure 1 Explanation of 2.5D approximation

of chemical kinetics, is solved. Each equation of RANS system can be represented in the following form:

$$
\frac{\partial a}{\partial t}+\frac{\partial F_{x}}{\partial x}+\frac{\partial F_{y}}{\partial y}+\frac{\partial F_{z}}{\partial z}=W
$$

Here, $a$ is the quantity of some physical parameter (mass, momentum, energy, turbulence parameters, or mass of the reacting mixture chemical component) per unit volume of gas; $F_{i}$ is the flux of $a$ value along $x_{i}$ axis $\left(x_{1} \equiv x, x_{2} \equiv y\right.$, and $x_{3} \equiv z$ ); and $W$ are the local sources of $a$.

Inner space of the duct is subdivided into following elementary volumes:

$$
V=\left[x-\frac{\Delta x}{2}, x+\frac{\Delta x}{2}\right] \times\left[y-\frac{\Delta y}{2}, y+\frac{\Delta y}{2}\right] \times\left[z^{-}(x, y), z^{+}(x, y)\right],
$$

$h_{z}(x, y)=z^{+}(x, y)-z^{-}(x, y)$ is the duct width in $z$ direction. One such element, corresponding to an arbitrary cell of computational grid $[x-\Delta x / 2, x+\Delta x / 2]$ $\times[y-\Delta y / 2, y+\Delta y / 2]$, is shown in Fig. 1. The 2.5D approximation is obtained, if one assumes that flow parameters in each element are constant along $z$ axis. Characteristics of $2.5 \mathrm{D}$ flow in this cell may be treated as the result of real 3D flow averaging along $z$ direction:

$$
a_{2.5 \mathrm{D}}(x, y) \approx \frac{1}{h_{z}(x, y)} \int_{z^{-}(x, y)}^{z^{+}(x, y)} a(x, y, z) d z
$$


After that, let us integrate Eq. (1) over the constant volume defined by Eq. (2), assuming flow parameters to be constant along $z$ direction and applying Gauss-Ostrogradsky theorem:

$$
\begin{aligned}
& \frac{\partial a}{\partial t} \Delta x \Delta y h_{z} \\
& +\left(F_{x}\left(x+\frac{\Delta x}{2}, y\right) h_{z}\left(x+\frac{\Delta x}{2}, y\right)-F_{x}\left(x-\frac{\Delta x}{2}, y\right) h_{z}\left(x-\frac{\Delta x}{2}, y\right)\right) \Delta y \\
& +\left(F_{y}\left(x, y+\frac{\Delta y}{2}\right) h_{z}\left(x, y+\frac{\Delta y}{2}\right)-F_{y}\left(x, y-\frac{\Delta y}{2}\right) h_{z}\left(x, y-\frac{\Delta y}{2}\right)\right) \Delta x \\
& +\left(F_{x}^{-} S_{x}^{-}+F_{x}^{+} S_{x}^{+}+F_{y}^{-} S_{y}^{-}+F_{y}^{+} S_{y}^{+}+F_{z}^{-} S_{z}^{-}+F_{z}^{+} S_{z}^{+}\right)=W \Delta x \Delta y h_{z} .
\end{aligned}
$$

Here, $\vec{S}^{+}=\left(S_{x}^{+} ; S_{y}^{+} ; S_{z}^{+}\right)$is the vector of outward normal to the volume element's front side (coordinates of this side center are $\left(x, y, z^{+}(x, y)\right)$ ), and $\vec{S}^{-}$ $=\left(S_{x}^{-} ; S_{y}^{-} ; S_{z}^{-}\right)$is the vector of outward normal to the volume element's back side (coordinates of this side center are $\left.\left(x, y, z^{-}(x, y)\right)\right)$. Magnitudes of these vectors are equal to the areas of corresponding sides; magnitudes of the components of these vectors are equal to the areas of projections of these sides on planes, perpendicular to coordinate axes. Therefore,

$$
\begin{aligned}
& S_{x}^{ \pm} \approx \mp \Delta y \cdot\left(z^{ \pm}\left(x+\frac{\Delta x}{2}, y\right)-z^{ \pm}\left(x-\frac{\Delta x}{2}, y\right)\right) ; \\
& S_{y}^{ \pm} \approx \mp \Delta x \cdot\left(z^{ \pm}\left(x, y+\frac{\Delta y}{2}\right)-z^{ \pm}\left(x, y-\frac{\Delta y}{2}\right)\right) ; \\
& S_{z}^{ \pm}= \pm \Delta x \cdot \Delta y .
\end{aligned}
$$

Then, let us substitute these relations into Eq. (4), divide it by $\Delta x \Delta y$, and consider the limit $\Delta x \rightarrow 0, \Delta y \rightarrow 0$ :

$$
\begin{aligned}
& \frac{\partial}{\partial t}\left(a h_{z}\right)+\frac{\partial}{\partial x}\left(F_{x} h_{z}\right)+\frac{\partial}{\partial y}\left(F_{y} h_{z}\right) \\
& \quad+\left(F_{x}^{-} \frac{\partial z^{-}}{\partial x}-F_{x}^{+} \frac{\partial z^{+}}{\partial x}+F_{y}^{-} \frac{\partial z^{-}}{\partial y}-F_{y}^{+} \frac{\partial z^{+}}{\partial y}+F_{z}^{+}-F_{z}^{-}\right)=W h_{z} .
\end{aligned}
$$

This is 2.5D analogue of Eq. (1). By writing Eq. (5) for $a=\rho, \rho u, \rho v, \rho E, \rho p_{k}^{t}$; and $\rho Y_{m}$, one gets the equation system for $2.5 \mathrm{D}$ analogue of $3 \mathrm{D}$ flow in the duct. Here and below, $\rho$ is the density; $u$ and $v$ are the velocity components along $x$ and $y$ axes, respectively; $E$ is the total energy per unit mass of gas:

$$
E=\frac{u^{2}+v^{2}}{2}+\bar{k}+\sum_{m=1}^{N_{\mathrm{sp}}} Y_{m} e_{m}(T)
$$


$p_{k}^{t}, k=1, \ldots, N_{\mathrm{turb}}$, are the parameters of the considered model of turbulence; $Y_{m}, m=1, \ldots, N_{\mathrm{sp}}$, are the mass fractions of the reactive mixture components; $\bar{k}$ is the averaged kinetic energy of turbulence; $e_{m}(T)$ is the internal energy of the $m$ th component of gas mixture; and $T$ is the temperature. In this work, $q-\omega$ turbulence model [9-11] is considered, for which $N_{\text {turb }}=2, p_{1}^{t} \equiv q \equiv \sqrt{\bar{k}}$ is the characteristic value of turbulent fluctuations of velocity, and $p_{2}^{t} \equiv \omega \equiv \bar{\varepsilon} / \bar{k}$ is the characteristic frequency of turbulent fluctuations $(\bar{\varepsilon}$ is the average rate of turbulent kinetic energy dissipation). Chemical kinetics model, consisting of $N_{\text {sp }}=9$ components $\left(\mathrm{H}, \mathrm{O}, \mathrm{OH}, \mathrm{H}_{2} \mathrm{O}, \mathrm{O}_{2}, \mathrm{H}_{2}, \mathrm{CO}, \mathrm{CO}_{2}\right.$, and $\left.\mathrm{N}_{2}\right)$ is used.

Essential moment in construction of $2.5 \mathrm{D}$ analogue of $3 \mathrm{D}$ flow in the duct is the way to determine the fluxes through lateral sides $F_{i}^{-}$and $F_{i}^{+}$that are placed in additional source terms in the left-hand side of Eq. (5). As well as in construction of quasi-1D ("1.5D") flow theories, these fluxes should be determined taking into account the real 3D flow structure near the duct walls.

Vector of fluxes along $x_{i}$ axis $\left(x_{1}=x, x_{2}=y\right.$, and $\left.x_{3}=z\right)$ on solid impermeable surface without slip can be represented as follows:

$$
\vec{F}_{i}^{ \pm}=\left[\begin{array}{c}
F_{i}^{ \pm}(\rho) \\
F_{i}^{ \pm}(\rho u) \\
F_{i}^{ \pm}(\rho v) \\
\left.F_{i}^{ \pm} \rho E\right) \\
F_{i}^{ \pm}\left(\rho p_{k}^{t}\right) \\
F_{i}^{ \pm}\left(\rho Y_{m}\right)
\end{array}\right]=\left[\begin{array}{c}
0 \\
p_{W} \delta_{x i}-\mu_{W} \frac{\partial V_{\tau}}{\partial n}\left(\tau_{x} n_{i}+\tau_{i} n_{x}\right) \\
p_{W} \delta_{y i}-\mu_{W} \frac{\partial V_{\tau}}{\partial n}\left(\tau_{y} n_{i}+\tau_{i} n_{y}\right) \\
-\frac{\mu_{W} C_{P}}{\operatorname{Pr}} \frac{\partial T}{\partial n} n_{i} \\
-\frac{\mu_{W}}{\operatorname{Pr}\left(p_{k}^{t}\right)} \frac{\partial p_{k}^{t}}{\partial n} n_{i} \\
0
\end{array}\right] .
$$

Here, $n$ is the component of unit vector of local outward normal to the duct wall $\vec{n}^{ \pm}(x, y)=\left(n_{x}^{ \pm} ; n_{y}^{ \pm} ; n_{z}^{ \pm}\right) ; V_{\tau} \equiv \vec{V} \cdot \vec{\tau}$ is the tangential-to-wall component of velocity (direction of $\vec{\tau}$ vector coincides with limit direction of velocity vector, when the point approaches to the wall); index "W" marks the values of parameters on the duct wall. Components of the unit outward normal to wall $\vec{n}^{ \pm}$can be expressed as follows:

$$
\begin{array}{r}
\vec{n}^{ \pm} \equiv\left(n_{x}^{ \pm}, n_{y}^{ \pm}, n_{z}^{ \pm}\right) \\
=\lim _{\Delta x \Delta y \rightarrow 0} \frac{\left(S_{x}^{ \pm} /(\Delta x \Delta y) ; S_{y}^{ \pm} /(\Delta x \Delta y) ; S_{z}^{ \pm} /(\Delta x \Delta y)\right)}{\sqrt{\left(S_{x}^{ \pm} /(\Delta x \Delta y)\right)^{2}+\left(S_{y}^{ \pm} /(\Delta x \Delta y)\right)^{2}+\left(S_{z}^{ \pm} /(\Delta x \Delta y)\right)^{2}}} \\
=\frac{\left(\mp \partial z^{ \pm} / \partial x ; \mp \partial z^{ \pm} / \partial y ; \pm 1\right)}{\sqrt{\left(\partial z^{ \pm} / \partial x\right)^{2}+\left(\partial z^{ \pm} / \partial y\right)^{2}+1}} .
\end{array}
$$

Components of unit vector $\vec{\tau}$, which is parallel to tangential velocity near wall, can be chosen on the basis of assumption that projection of the tangen- 
tial velocity vector on $(x ; y)$ plane is codirectional to velocity vector of $2.5 \mathrm{D}$ $\left(z\right.$-averaged) flow that has components $(u(x, y), v(x, y)):\left(\tau_{x}, \tau_{y}\right) \|(u, v)$. From condition $\vec{\tau} \cdot \vec{n}=0$, one may find $\tau_{z}=-\left(\tau_{x} n_{x}+\tau_{y} n_{y}\right) / n_{z}$. Finally, let us normalize $\vec{\tau}$ vector and get:

$$
\vec{\tau}^{ \pm} \equiv\left(\tau_{x}^{ \pm}, \tau_{y}^{ \pm}, \tau_{z}^{ \pm}\right)=\frac{\left(u ; v ; u \partial z^{ \pm} / \partial x+v \partial z^{ \pm} / \partial y\right)}{\sqrt{u^{2}+v^{2}+\left(u \partial z^{ \pm} / \partial x+v \partial z^{ \pm} / \partial y\right)^{2}}}
$$

In determination of wall fluxes, let us use the model of $3 \mathrm{D}$ flow consisting of inviscid core, where the pressure is constant along $z$ direction, and of boundary layers, where the flow is decelerated to zero velocity. Due to the fact that the pressure is practically constant across boundary layer, let us take wall pressure to be equal to pressure in the inviscid core and to $z$-averaged value of pressure (i.e., with pressure in $2.5 \mathrm{D}$ analogue of this $3 \mathrm{D}$ flow). In this case, the wall pressure $p_{W}$ is equal to pressure of $2.5 \mathrm{D}$ flow in current spatial element $p(x, y)$.

Molecular diffusive fluxes of momentum, heat, and turbulence parameters in the direction of the local normal to the wall (in Eq. (6), they have form $\left.\mu_{W} \partial f / \partial n\right)$ are determined by local structure of boundary layer.

In $2.5 \mathrm{D}$ calculation, boundary layers appear only near upper and lower contours of the duct. Let us estimate the local values of $\mu_{W} \partial f / \partial n$ through linear interpolation in $y$ between upper and lower contours of the duct. Therefore, let us take

$$
\left.\begin{array}{rl}
p_{W}(x, y) & =p(x, y) \\
\left.\left(\mu_{W} \frac{\partial f}{\partial n}\right)\right|_{(x, y)}= & \frac{\left.\left(\mu_{W} \partial f / \partial n\right)\right|_{\left(x, y^{+}(x)\right)}\left(y-y^{-}(x)\right)}{y^{+}(x)-y^{-}(x)} \\
& \quad+\frac{\left.\left(\mu_{W} \partial f / \partial n\right)\right|_{\left(x, y^{-}(x)\right)}\left(y^{+}(x)-y\right)}{y^{+}(x)-y^{-}(x)}
\end{array}\right\}
$$

where $y^{+}(x)$ and $y^{-}(x)$ are the coordinates of the duct contours in the computational domain. It is necessary to underline that $n$-direction is determined by local outward normal to the duct wall; it is different for upper contour of duct, for its lower contour, and for current point of computational domain $(x, y)$.

Other terms of Eq. (5) will be calculated using the same formulas as the corresponding terms in the initial equation system of $3 \mathrm{D}$ flow. But we shall substitute parameters of $2.5 \mathrm{D}$ flow (in fact, $z$-averaged parameters of real flow) into these formulas. Naturally, this approach to description of 3D flow is approximate, because we assume that $z$-averaged fluxes and sources in equation of gas motion can be calculated by usual formulas, where $z$-averaged gas parameters 
are substituted. Average of nonlinear function does not coincide with the value of this function after substitution of average values of its arguments. Therefore, fluxes and source terms will be determined with some errors. These errors, together with errors of approximate formulas (7), constitute the inaccuracy of $2.5 \mathrm{D}$ approximation.

The most important target function in simulation of flow in combustion chamber is the longitudinal force $R$. This is $x$-component of the integral force that is applied to the duct surface. By definition, this force is equal to integral of momentum $x$-component over the duct surface. Accordingly, this force should be calculated as follows:

$$
\begin{aligned}
R=\int_{y=y^{-}(x)}\left(F_{x}(\rho u) h_{z} d l_{x}+F_{y}(\rho u) h_{z} d l_{y}\right) & +\int_{y=y^{+}(x)}\left(F_{x}(\rho u) h_{z} d l_{x}+F_{y}(\rho u) h_{z} d l_{y}\right) \\
& +\iint\left(F_{x}^{-}(\rho u) \frac{\partial z^{-}}{\partial x}-F_{x}^{+}(\rho u) \frac{\partial z^{+}}{\partial x}\right. \\
& +F_{y}^{-}(\rho u) \frac{\partial z^{-}}{\partial y}-F_{y}^{+}(\rho u) \frac{\partial z^{+}}{\partial y} \\
& \left.+F_{z}^{+}(\rho u)-F_{z}^{-}(\rho u)\right) d s(x, y)
\end{aligned}
$$

In this formula, $F_{i}(\rho u)$ is the flux of momentum $x$-component along $x_{i}$ axis. The first integral is calculated over the lower contour of the duct and the second integral - over its upper contour. In these integrals, $\overrightarrow{d l}=\left(d l_{x} ; d l_{y}\right)$ is the vector with magnitude equal to the length of the duct contour element, this vector is codirectional with outward normal to the contour; $h_{z} d l_{x}$ is the projection of the duct surface part, corresponding to this contour element, on the plane that is perpendicular to $x$ axis; and $h_{z} d l_{y}$ is its projection on the plane that is perpendicular to $y$ axis. In practice, sides of near-boundary computational cells are used as the contour elements.

It is worth to note that $h_{z} \neq 0$, only if the duct has upper or lower walls parallel to the $z$ axis. In the example, which is shown in Fig. 1, such walls are absent, and first two integrals in Eq. (8) are equal to zero.

The third (surface) integral is taken over the inner part of the computational domain (of the duct projection on the $(x ; y)$ plane). In this integral, $d s(x, y)$ is the area of grid cell; and $\left(\partial z^{-} / \partial x\right) d s,\left(\partial z^{-} / \partial y\right) d s$, and $(-d s)$ are the components of the vector of the duct back surface element that corresponds to this element of computational domain. The length of this vector is equal to the area of the surface element and its direction coincides with direction of outward normal to surface; and $\left(-\left(\partial z^{+} / \partial x\right) d s\right),\left(-\left(\partial z^{-} / \partial y\right) d s\right)$, and $d s$ are the components of the vector of the duct front surface element that corresponds to this grid cell. 


\section{VERIFICATION OF CODE FOR QUASI-TWO-DIMENSIONAL CALCULATIONS}

New possibility to simulate flows in ducts in $2.5 \mathrm{D}$ approximation has been realized in scientific code SOLVER3 [12] that is intended for calculation of 2D flows of multicomponent gas on the basis of RANS equations (previously, this code allowed to calculate only planar and axisymmetric flows). This section describes the test cases that were used to verify the module of SOLVER3 code where the new technique was realized.

The first test is based on the fact that the new approach to simulation of $3 \mathrm{D}$ flows generalizes the classical theory of quasi-1D flows, which is described, e.g., in [13]. In particular, theory of Laval nozzle is based on the equations of quasi-1D flow.

Theory of Laval nozzle considers a duct with given law of area variation $F(x)$. Flow in the duct is assumed to be stationary and inviscid. Quasi-1D ("1.5D") analogue of this flow can be obtained, if one assumes that flow parameters are constant in each cross section of the duct, but vary from one section to another. In fact, it means that the parameters of real flow are averaged over the duct section.

Equations of the Laval nozzle theory can be considered as particular case of $2.5 \mathrm{D}$ flow equations (5). To deduce these equations from (5), the $2.5 \mathrm{D}$ theory assumption about constant flow parameters along $z$ axis should be supplied with assumptions that stationary flow of single-component perfect gas is considered in a duct that has constant width $\Delta y=$ const in $(x, y)$ plane, while its lateral width is changed according to law

$$
h_{z} \equiv z^{+}(x)-z^{-}(x)=\frac{F(x)}{\Delta y} .
$$

With the use of these additional assumptions, Eqs. (5) can be rewritten as follows:

$$
\begin{gathered}
\frac{d}{d x}\left(\vec{F}_{x} h_{z}\right)+\left(\vec{F}_{x}^{-} \frac{d z^{-}}{d x}-\vec{F}_{x}^{+} \frac{d z^{+}}{d x}\right)=0 ; \\
\vec{F}_{x}=\left[\begin{array}{c}
\rho u \\
\rho u^{2}+p \\
\rho u E+p u
\end{array}\right] ; \quad \vec{F}_{x}^{+}=\vec{F}_{x}^{-}=\left[\begin{array}{c}
0 \\
p_{W} \\
0
\end{array}\right] .
\end{gathered}
$$

Taking $p_{W}=p$ and $h_{z}(x)=F(x) / \Delta y$, after transformations, one can obtain the classical equations of Laval nozzle theory:

$$
\rho u \frac{d u}{d x}=-\frac{d p}{d x} ; \quad \rho u F=\text { const } ; \quad \frac{u^{2}}{2}+\frac{\gamma}{\gamma-1} \frac{p}{\rho}=\text { const } .
$$


Ordinary differential equation with two closing relations (9) can be solved numerically with very high accuracy and can be used as reference. Calculation of flow in duct with $\Delta y=$ const, $h_{z}(x)=F(x) / \Delta y$ on the basis of Eqs. (5) should give the solution that coincides with the reference solution within the approximation errors.

As the second test for the verification of $2.5 \mathrm{D}$ code, calculations of viscous turbulent flow in axisymmetric duct with supersonic flow at the entrance have been used. In the $(x, y)$ plane, uniform grid, containing 398 cells along the duct and 70 cells across the duct, have been constructed. Uniform flow of air with specific heat ratio $\gamma=1.4$, with Mach number $\mathrm{M} \approx 2.9$, with stagnation parameters $p_{0} \approx 15$ atm and $T_{0}=2150 \mathrm{~K}$ and with turbulence parameters $q=16 \mathrm{~m} / \mathrm{s}$ and $\omega=2000 \mathrm{~Hz}$. On the duct walls, boundary condition of the class "wall functions" $[12,14]$ was used (it allows to avoid extreme compression of grid to no-slip walls). Parameters in the exit section of the duct were taken from the near-boundary cells.

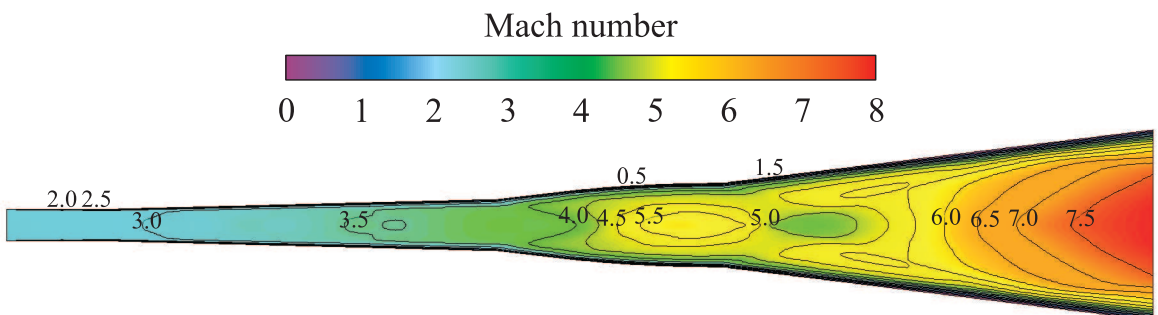

(a)

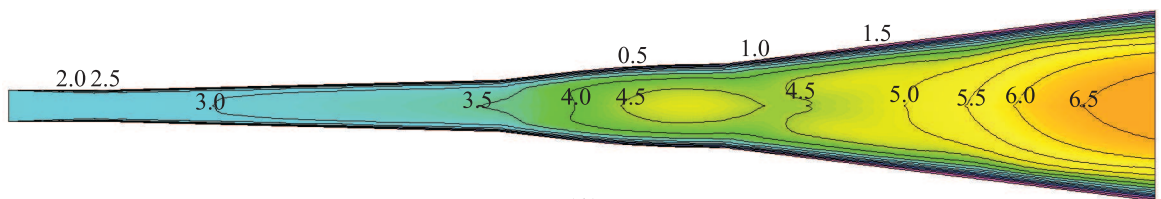

(b)

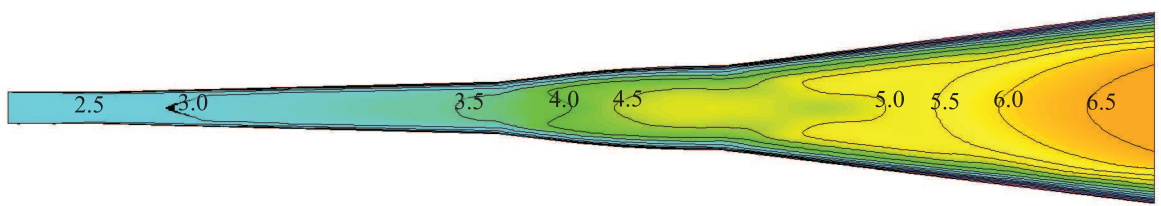

(c)

Figure 2 Fields of Mach number, obtained in calculations of axisymmetric duct: $(a)$ axisymmetric calculation; $(b)$ averaging of axisymmetric flowfield along $z$ axis; and (c) $2.5 \mathrm{D}$ calculation 


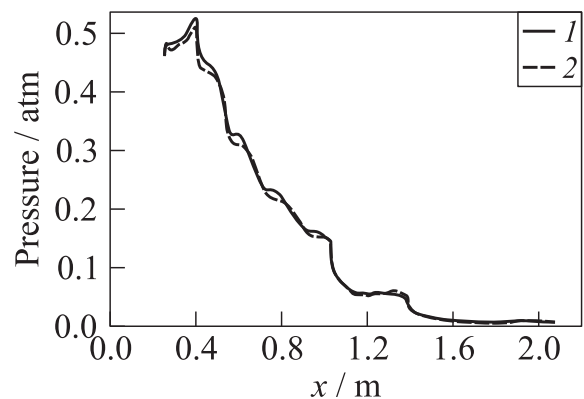

(a)

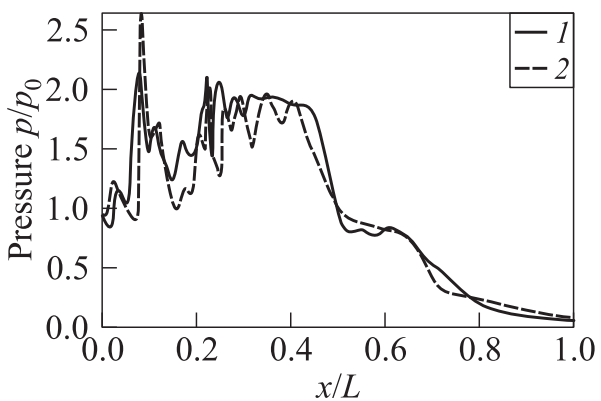

(b)

Figure 3 Pressure distributions along the wall: $(a)$ comparison of axisymmetric (1) and 2.5D (2) calculations of axisymmetric duct; and (b) comparison of 3D (1) and $2.5 \mathrm{D}(2)$ calculations of scramjet combustor

In Fig. 2, three fields of Mach number in the axisymmetric duct are compared:

(1) reference field obtained by solution of axisymmetric flow equations;

(2) field obtained by averaging of the reference field along $z$ coordinate (see formula (3)); and

(3) result of the same duct calculation in $2.5 \mathrm{D}$ approximation.

One can see that results of $2.5 \mathrm{D}$ calculation are close to the parameters, obtained by averaging of the reference field along the lateral coordinate. Minor differences of these fields are resulted from the presence of 3D stationary wave structures in supersonic flow. Such structures cannot be reproduced correctly, if flow parameters are considered to be constant along $z$ axis. Thickness of boundary layer in $2.5 \mathrm{D}$ calculation is higher than in the axisymmetric calculation. But averaging of axisymmetric field along $z$ gives boundary layer of practically the same thickness as in the $2.5 \mathrm{D}$ calculation. Figure $3 a$ compares the pressure distributions along the duct walls, obtained in these calculations. The $2.5 \mathrm{D}$ calculation predicts the longitudinal distribution of loads on the duct walls with good accuracy.

\section{CALCULATIONS OF FLOW IN COMBUSTOR OF HIGH-SPEED AIRCRAFT}

A 2.5D approach has been applied to parametrical calculations of flow in the combustion chamber of a scramjet with hydrogen fuel for a hypothetic supersonic 


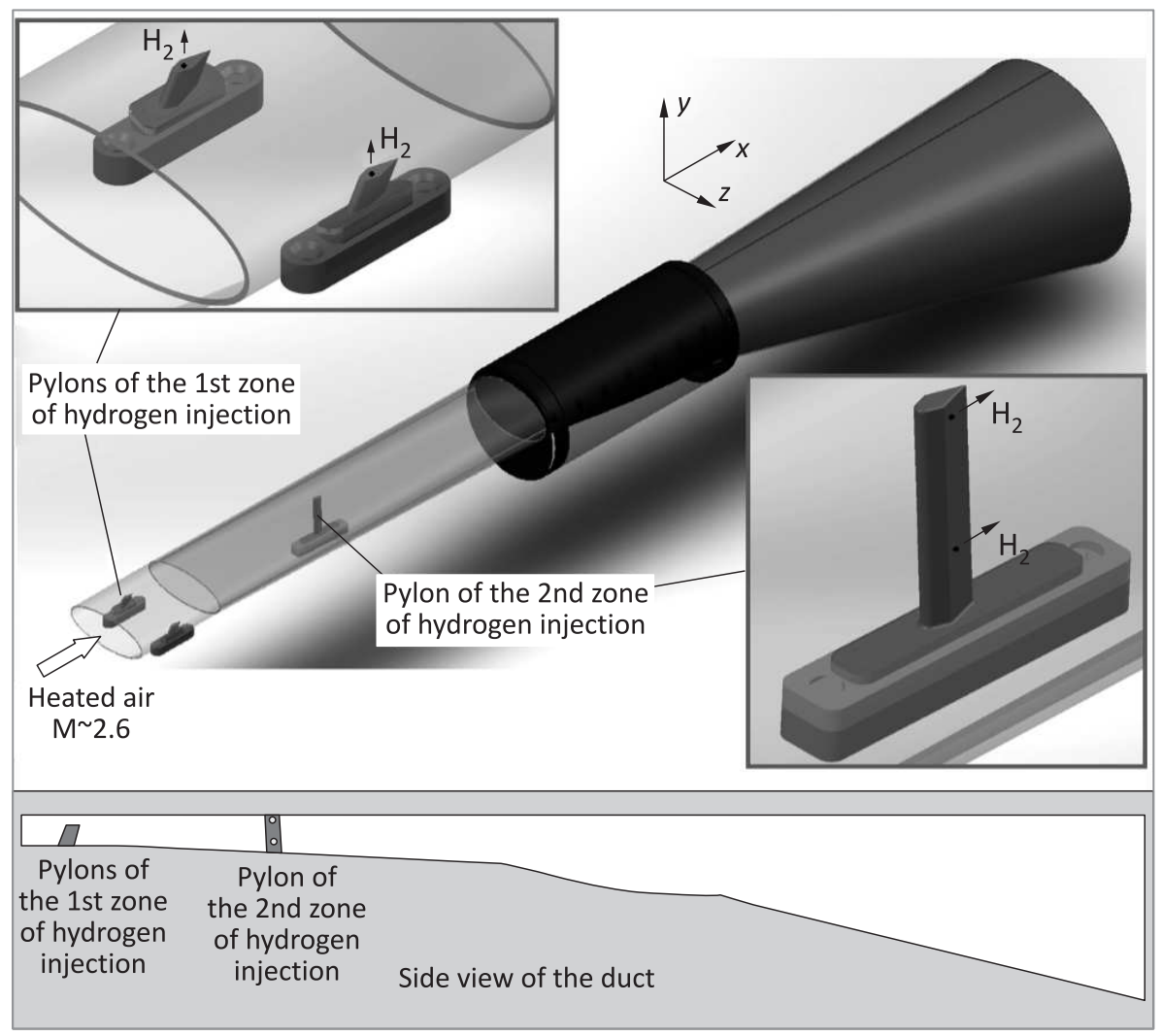

Figure 4 Geometry of scramjet combustion chamber

civil aircraft [15-18] that is studied in TsAGI in the framework of the HEXAFLYINT international project. It is a duct with elliptical sections with two zones of fuel injection (Fig. 4). In the 1st zone, hydrogen is injected upwards from two short pylons, while in the 2nd zone, in the flow direction from several holes in a high pylon placed in the symmetry plane of the duct. At the entrance of combustor, there is inflow of air heated by a flame heater and enriched with oxygen (to get the same mass fraction of oxygen as in air). Mach number in the flow inviscid core $\mathrm{M} \sim 2.6$, pressure is about $0.5 \mathrm{~atm}$. Temperature in the inviscid core at the entrance is close to $1200 \mathrm{~K}$ but temperature of the injected jets of hydrogen is equal to $163 \mathrm{~K}$ only.

Initially, preliminary 3D calculation has been performed with hydrogen injection, but without chemical reactions. The 3D calculations were performed using scientific code ZEUS-S3pp that is one component of EWT-TsAGI software 


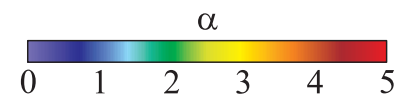

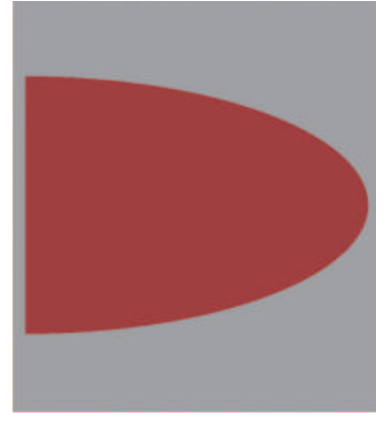

(a)

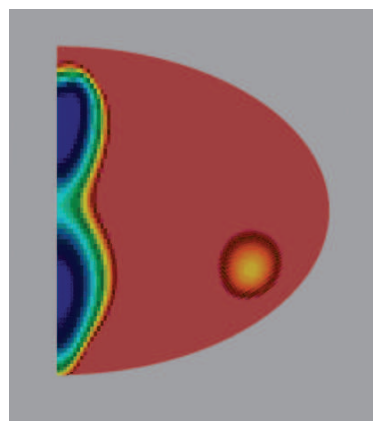

(d)

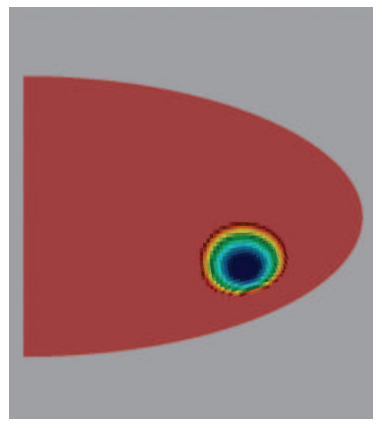

(b)

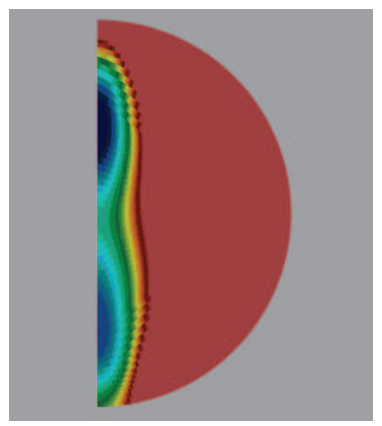

(e)

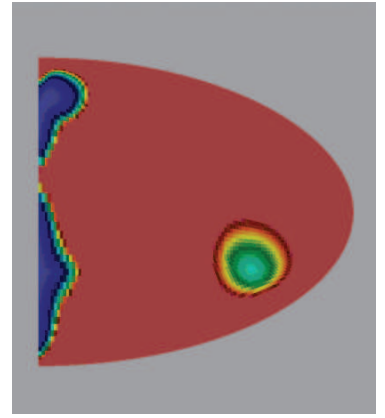

(c)

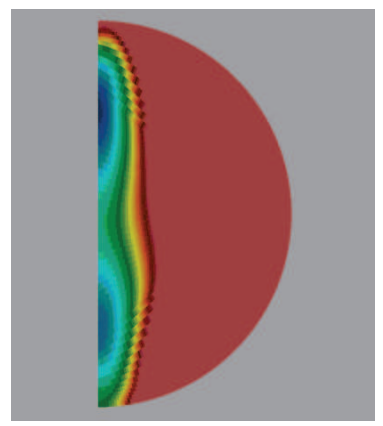

$(f)$

Figure 5 Fields of oxidizer excess ratio in several cross sections of combustor, obtained in preliminary $3 \mathrm{D}$ calculation without combustion: (a) $x / L=0 ;$ (b) $0.14 ;$ (c) 0.25 ; (d) $0.41 ;(e) 0.69$; and $(f) x / L=1$

package [19]. Calculation has been performed for the variant of fuel injection where $10 \%$ of the total hydrogen mass-flow rate were injected through each pylon of the 1st injection zone and the rest $80 \%$ were injected through the holes of the 2 nd injection zone pylon (scheme of fuel injection: $10 \%-10 \%-80 \%$ ).

In Fig. 5, the fields of oxidizer excess ratio, obtained in this calculation in several cross sections of the chamber, are shown. One can see that cross sections of fuel jets, injected from the central pylon, are spread in vertical direction. It is resulted from the fact that injected hydrogen enters into the wake past the central pylon. In the case of greater part of mass-flow rate through the 1st injection zone pylons, cross sections of jets from the 1st injection zone pylons should also be spread in vertical direction due to the upward injection of fuel. Therefore, flow 


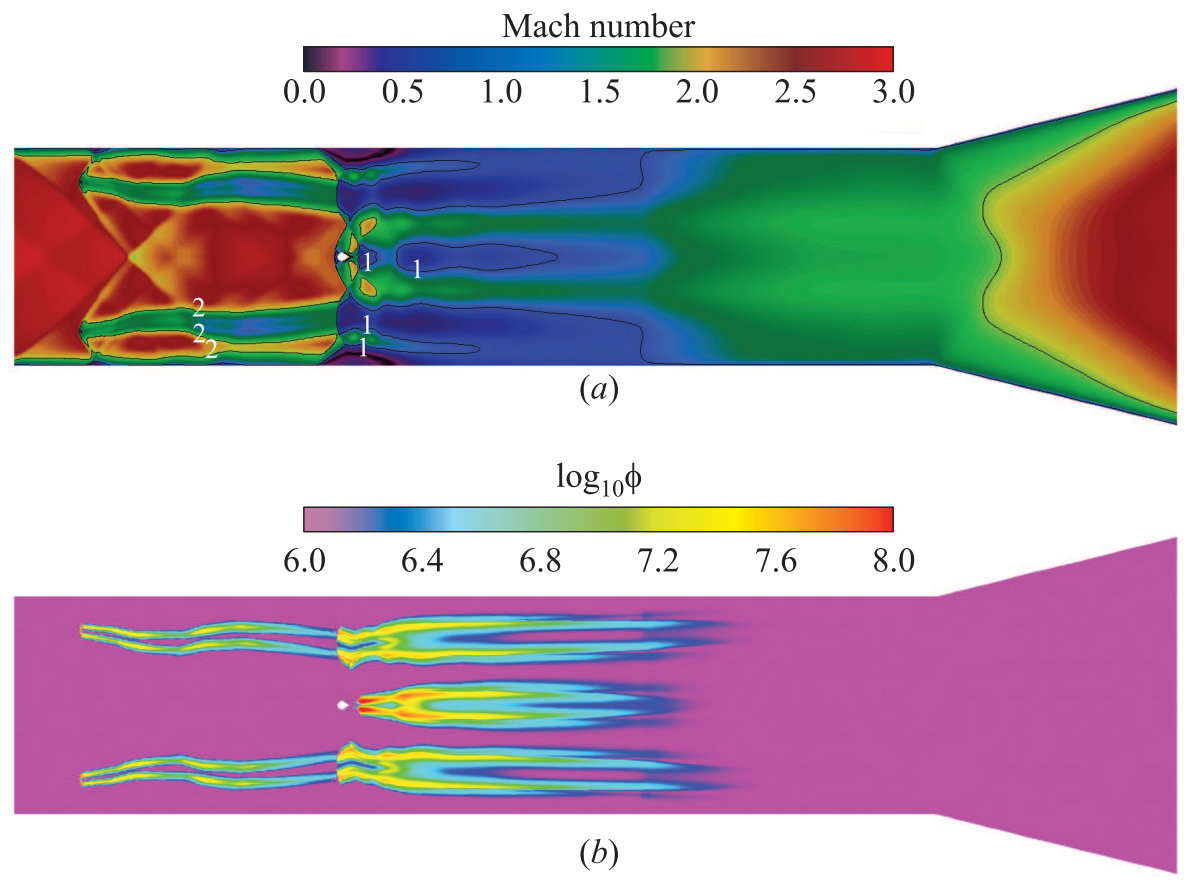

Figure 6 Fields of Mach number $(a)$ and decimal logarithm of heat production rate $(b)$, obtained in $2.5 \mathrm{D}$ calculations for the fuel injection scheme $30 \%-30 \%-40 \%$

parameters vary along $y$ axis weaker than along the $z$ axis. Consequently, there is sense to perform $2.5 \mathrm{D}$ calculations in the $(x ; z)$ plane instead of $(x ; y)$ plane. The $2.5 \mathrm{D}$ calculations in $(x ; y)$ plane give a picture of fuel mixing with air that differs principally from the picture obtained in $3 \mathrm{D}$ calculations. So, below, only $2.5 \mathrm{D}$ calculations in $(x ; z)$ plane will be considered. These calculations have been performed with finite-rate chemical reactions.

In Fig. 6a, the typical Mach number field, obtained in calculation for the fuel injection scheme $30 \%-30 \%-40 \%$, is shown. To demonstrate details, only a part of combustor is shown in this figure, and scale along the duct width is increased. This field shows that oblique shock waves arise ahead of the fuel jets, injected from the 1st injection zone pylons. At the place of their interaction with the wall, small separation of boundary layer arises. More intense shock wave is formed ahead the blunt leading edge of the central pylon. Its interaction with boundary layers on the duct walls leads to formation of separation zones of larger size. Oblique shocks, produced by these separations, intersect with leading shock wave from the central pylon in the region of jets from the 1st 
fuel injection zone, with lower Mach number. As a result, these shocks intersect irregularly, with formation of Mach disks, curved due to the flow inhomogeneity in the hydrogen jets. Behind the Mach disks, there are regions of subsonic flow. Further downstream, there are additional subsonic zones formed due to following intersections of shock waves.

Figure $6 b$ demonstrates for the same calculation the field of decimal logarithm of dimensional rate $\phi$ of heat release per unit length along a streamline:

$$
\phi(x, y)=(\rho|\vec{V}|)^{-1} \sum_{k=1}^{N_{\mathrm{sp}}} h_{k} m_{k} \sum_{l=1}^{N_{r}} v_{k l} W_{l} \quad[\mathrm{~J} /(\mathrm{kg} \cdot \mathrm{m})]
$$

where $N_{\mathrm{sp}}$ is the number of mixture components; $N_{r}$ is the number of reactions; $W_{l}$ is the molar rate of the $l$ th reaction; $v_{k l}$ is the stoichiometric coefficient of the $k$ th component in the $l$ th reaction; $m_{k}$ is the molecular weight of the $k$ th component; $h_{k}(T)$ is its static enthalpy (per unit mass); $\rho$ is the mixture density; and $|\vec{V}|$ is the magnitude of velocity vector. Derivation of formula (10) is given in [20]. Field of this parameter shows that in the jets of the 1st fuel injection zone, a weak heat release proceeds initially only in the outer regions of the jets (because of low temperature of hydrogen).

Essential heat release starts only downstream from the 2nd hydrogen injection zone: growth of pressure and of temperature in shock-wave structures accelerates the reaction and decrease of velocity in this region leads to longer residence of fuel in the reaction zone. Downstream from the 2 nd fuel injection zone, combustion proceeds in the regions of subsonic or transonic flow. Curved leading shock wave ahead of the central pylon and curved Mach disks and, also, boundary layer separations generate vorticity and become strong generators of turbulence. Growth of turbulence enhances combustion downstream from the 2nd hydrogen injection zone through the transport of heat from combustion zones to cold flow regions and through the transport of reagents to combustion zones. In the separations on the walls, there is no combustion because of the absence of fuel. Combustion practically stops at considerable distance upstream from the section, where the duct width begins to grow. When the heat release stops, the Mach number starts to grow. This effect is related with growth of the duct area (height of the duct increases along $x$ axis as it may be seen from the side view of the duct in Fig. 4); in addition, the turbulent diffusion carries heat across the duct and diminishes the average temperature of flow, enhancing increase of Mach number.

To determine an optimal proportion between mass-flow rates of hydrogen injected in the 1 st and $2 \mathrm{nd}$ zones, $2.5 \mathrm{D}$ calculations for the following variants have been performed: $10 \%-10 \%-80 \%$ (10\% of total mass-flow rate of hydrogen through the 1st zone pylons and 80\% - through the central pylon), 20\%-20\%$60 \%, 25 \%-25 \%-50 \%, 30 \%-30 \%-40 \%, 33 \%-33 \%-34 \%, 40 \%-40 \%-20 \%$, and $50 \%-$ 
Temperature / K

\begin{tabular}{rrrrrr}
\hline 1400 & 1600 & 1800 & 2000 & 2200 & 2400
\end{tabular}

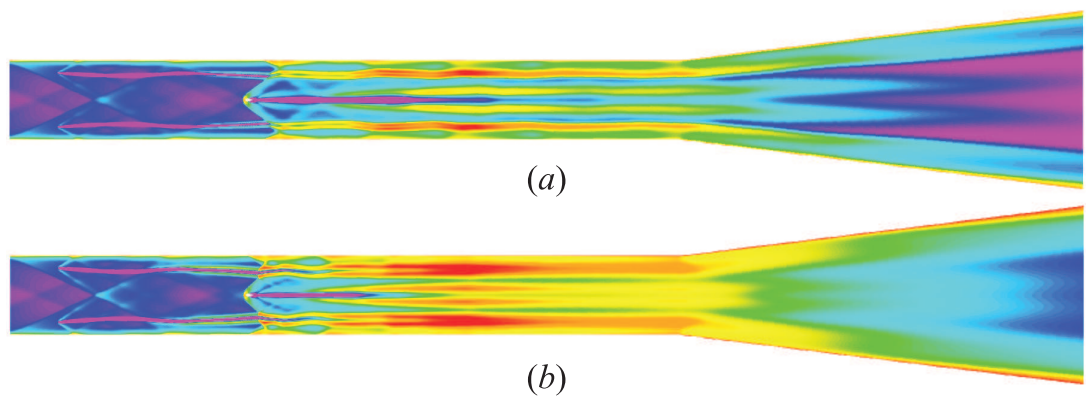

(b)

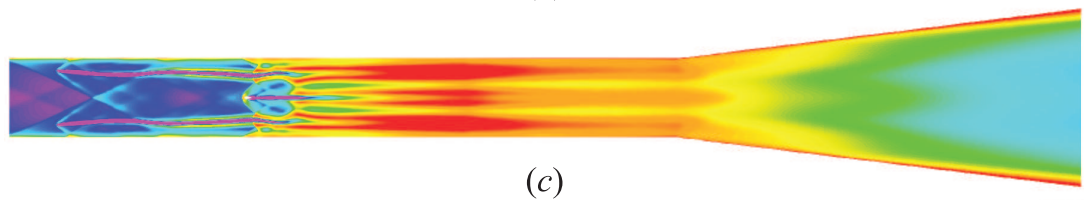

(c)

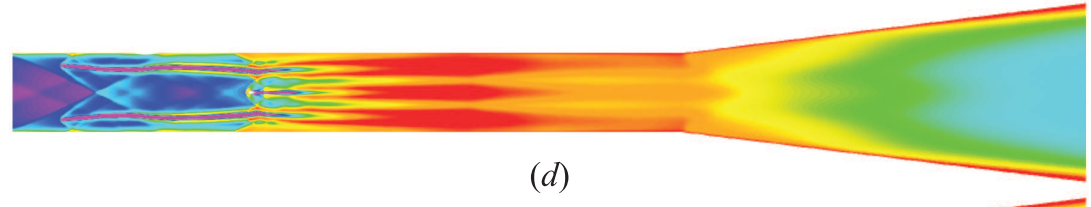

$(d)$

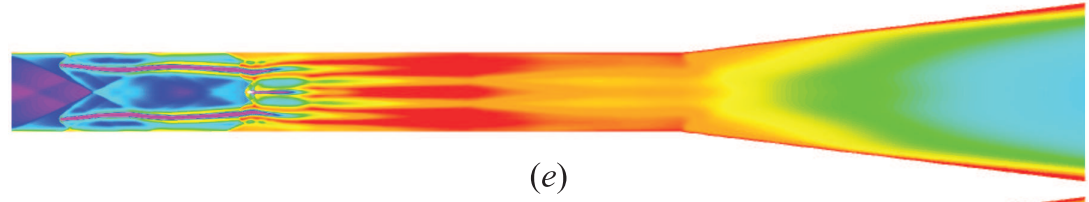

(e)
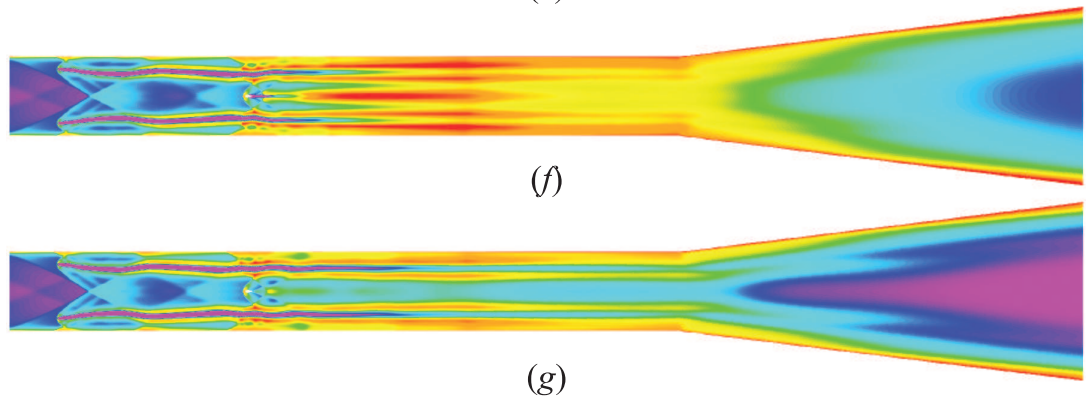

Figure 7 Fields of static temperature obtained in 2.5D calculations at different relations between hydrogen mass-flow rates in the 1st and 2nd injection zones: (a) 10\%$10 \%-80 \%$; (b) 20\%-20\%-60\%; (c) 25\%-25\%-50\%; (d) 30\%-30\%-40\%; (e) 33\%-33\%$34 \%$; $(f)$ 40\%-40\%-20\%; and $(g) 50 \%-50 \%-0 \%$ 
$50 \%-0 \%$. For each calculation, the integral longitudinal force applied to the chamber walls was determined by formula (8).

The best thrust characteristics have been obtained for the fuel injection scheme $30 \%-30 \%-40 \%$ and $33 \%-33 \%-34 \%$. Analysis of flow fields obtained in $2.5 \mathrm{D}$ calculations has allowed to explain this result.

In Fig. 7, the static temperature fields obtained in all calculations are shown. Because of low temperature of the injected hydrogen, in all schemes of fuel injection, the combustion upstream from the central pylon is possible only in outer regions of hydrogen jets, not inside these jets. Core of lateral jets remains to be too cold along the whole combustor in the scheme $50 \%-50 \%-0 \%$, while the core of central jet is cold throughout in the scheme $10 \%-10 \%-80 \%$. From the scheme 20\%-20\%-60\%, core of central jet has enough time to start burning. But full development of combustion in the whole core of the central jet becomes possible only in the schemes $30 \%-30 \%-40 \%$ and $33 \%-33 \%-34 \%$. In the case of scheme $30 \%-30 \%-40 \%$, the most uniform distribution of heat across the duct is achieved and inner thrust of combustor is close to maximum. In the scheme $33 \%-33 \%-34 \%$, heat release in the central jet is less due to lower quantity of fuel and in the scheme $40 \%-40 \%-20 \%$, the heat release in lateral jets also begins to diminish because of insufficient heating (thickness of jets increases with the growth of mass-flow rate).
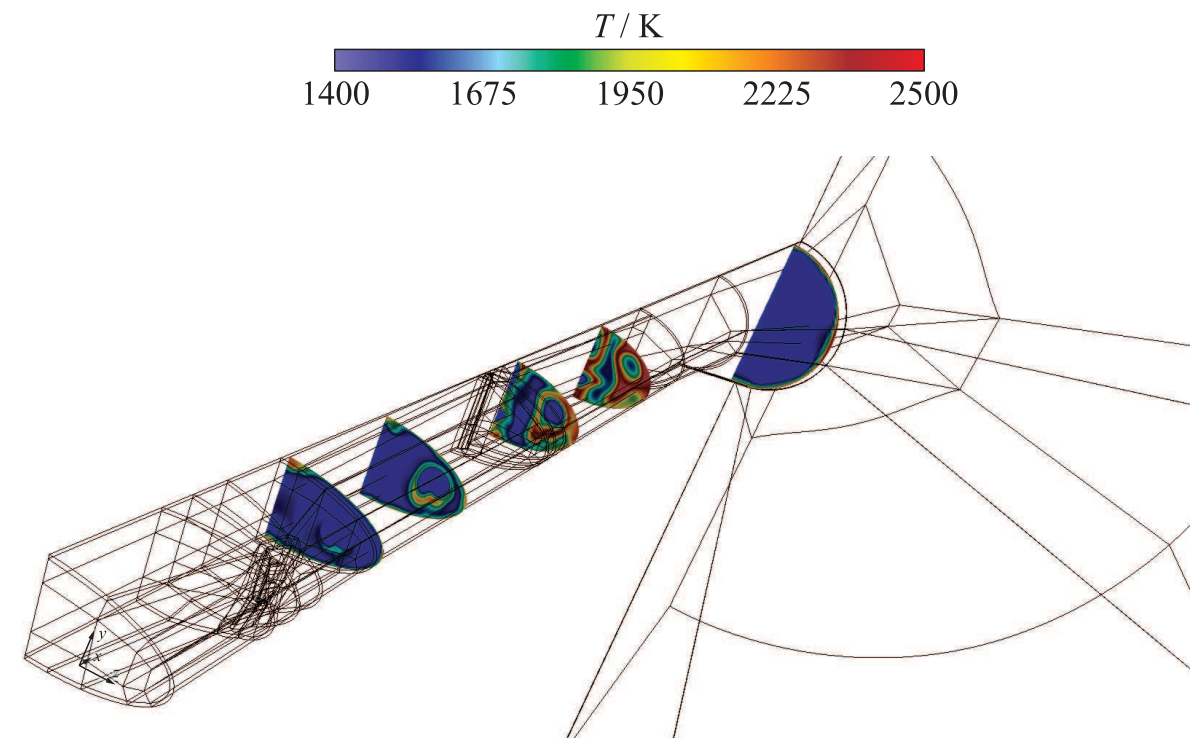

Figure 8 Three-dimensional view of combustion chamber: block structure of computational grid and fields of temperature in several cross sections 

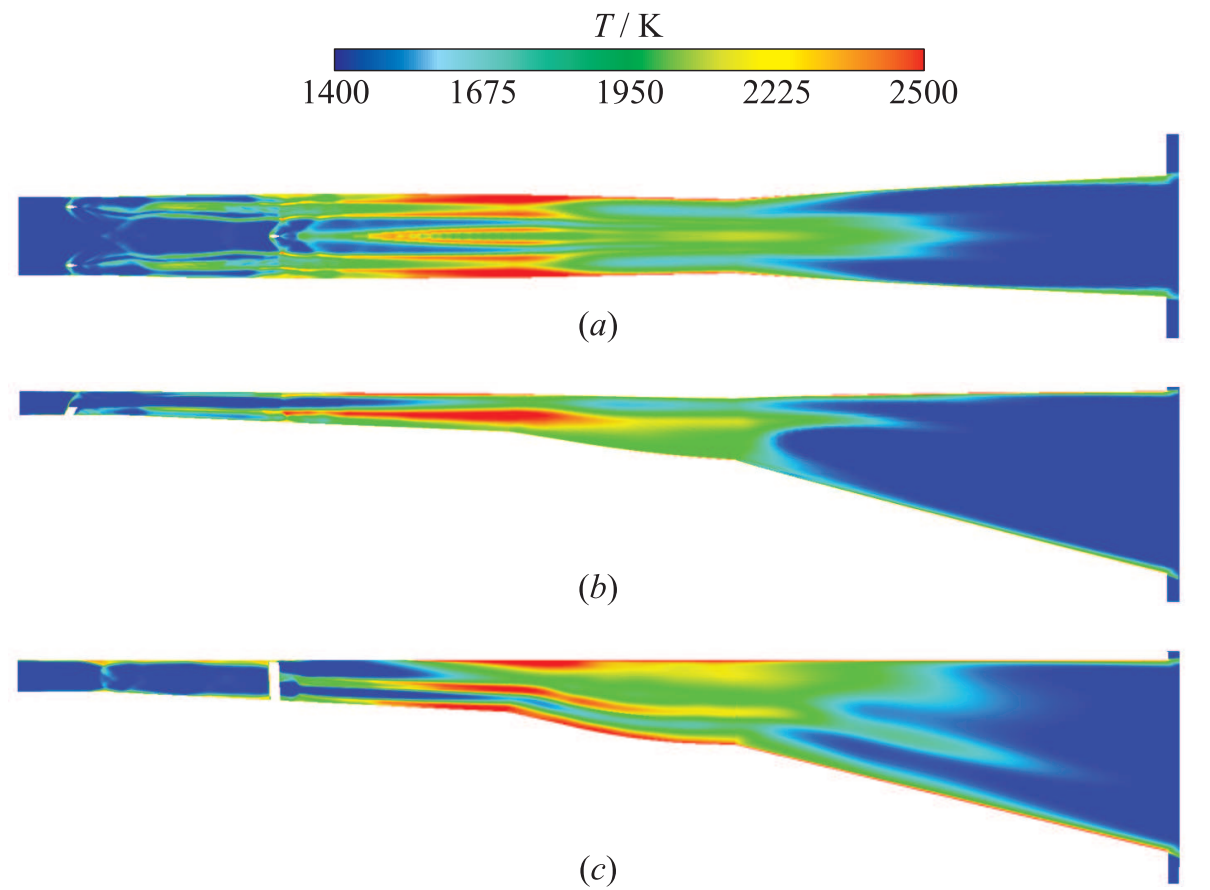

Figure 9 Fields of temperature obtained in 3D calculation for the fuel injection scheme 30\%-30\%-40\%: (a) one horizontal section; (b) vertical section through the pylon of the 1st injection zone; and $(c)$ vertical section through the pylon of the 2nd injection zone.

When in $2.5 \mathrm{D}$ calculations the most attractive regimes of flow in combustor had been found, 3D calculations of these regimes have been performed. In Fig. 8, the block structure of computational grid for 3D calculation and fields of temperature in several cross sections of combustor are shown.

Figure 9 shows the fields of temperature in the combustor sections by horizontal (Fig. 9a) and vertical (Figs. $9 b$ and $9 c$ ) planes. The presented data correspond to the fuel injection scheme $30 \%-30 \%-40 \%$.

Figure $9 a$ demonstrates that it is possible to find horizontal section of the combustor, where the flow field is qualitatively analogous to fields, obtained in $2.5 \mathrm{D}$ calculations. But flow structure in $3 \mathrm{D}$ calculation is naturally more complex. For example, it is impossible to reproduce the region of slow flow in the wake after the pylons of the 1st injection zone. Moreover, the injected jets of hydrogen are placed at different heights. As a result, maximum of temperature that can be seen in $2.5 \mathrm{D}$ calculation at the duct symmetry plane is absent in the horizontal section in Fig. $9 a$. This maximum is reached on the outer boundary 
of the upper central jet and it is placed below the plane of Fig. 9a. One can see this maximum in the combustor section by the vertical symmetry plane (see Fig. 9c).

In Fig. $3 b$, the distribution of static pressure along centerline of the duct upper wall, obtained in 3D calculation for the fuel injection scheme $30 \%^{-}-30 \%-$ $40 \%$, is compared with the static pressure distribution along the centerline of computational domain in the corresponding 2.5D calculation. Agreement is quite satisfactory, especially if one takes into account that the plot from $3 \mathrm{D}$ calculation is obtained with local values of pressure, whereas in $2.5 \mathrm{D}$ calculation, the pressure is averaged along $y$ axis.

\section{CONCLUDING REMARKS}

Parametrical study, described in section 4, would be impossible in the framework of quasi-1D theories, and 3D calculations would require too big resources of computer time and memory. In addition, physical analysis of flow structure obtained in $2.5 \mathrm{D}$ calculations is much more simple than in the $3 \mathrm{D}$ case.

The 2.5D approach is approximate way of flow analysis. It cannot take into account all features of $3 \mathrm{D}$ flows but it provides much more information about flow physics than the quasi-1D calculations. That is why, it may be recommended for use at the stage of preliminary design of combustion chambers. The 2.5D calculations can allow to make preliminary choice of most valuable variants of geometry and of flow regime and to diminish essentially the quantity of expensive and prolonged $3 \mathrm{D}$ calculations (which are, of course, necessary at the final stage of the combustor design).

\section{ACKNOWLEDGMENTS}

This work was performed within the 'High-Speed Experimental Fly VehiclesInternational' project fostering International Cooperation on Civil High-Speed Air Transport Research. HEXAFLY-INT, coordinated by ESA-ESTEC, is supported by the EU within the 7th Framework Programme Theme 7 Transport, Contract No. ACP0-GA-2014-620327. Further info on HEXAFLY-INT can be found at http://www.esa.int/hexafly-int.

\section{REFERENCES}

1. Wild, J., J. Brezillon, O. Amoignon, J. Quest, F. Moens, and D. Quagliarella. 2007. Advanced high-lift design by numerical methods and wind tunnel verification within the European Project EUROLIFT II. AIAA Paper No. 2007-4300. 18 p. 
2. Kanazaki, M., T. Imamura, S. Jeong, and K. Yamamoto. 2008. High-lift wing design in consideration of sweep angle effect using Kriging model. AIAA Paper No. 2008-175. 16 p.

3. Dandois, J., V. Brunet, P. Molton, J. C. Abart, and A. Lepage. 2010. Buffet control by means of mechanical and fluidic vortex generators. AIAA Paper No. 2010-4975. $14 \mathrm{p}$.

4. Li, C., S. Zhu, Y.L. Xu, and Y. Xiao. 2013. 2.5D large eddy simulation of vertical axis wind turbine in consideration of high angle of attack flow. Renew. Energ. 51:317-330.

5. Narayan, J. P. 1998. 2.5-D numerical simulation of acoustic wave propagation. Pure Appl. Geophys. 151(1):47-61.

6. Desquesnes, G. 2008. Euler equations in perturbation 2.5-D: A new system for acoustic modal propagation. AIAA Paper No. 2008-2822. 11 p.

7. Chen, Q., R. Temam, and J. J. Tribbia. 2008. Simulations of the 2.5D inviscid primitive equations in a limited domain. J. Comput. Phys. 227(23):9865-9884.

8. Kalise, D., and I. Lie. 2012. Modeling and numerical approximation of a 2.5D set of equations for mesoscale atmospheric processes. J. Comput. Phys. 231(21):72747298.

9. Coakley, T. J. 1983. Turbulence modeling methods for the compressible NavierStokes equations. AIAA Paper No. 83-1693. 14 p.

10. Coakley, T. J., and T. Hsieh. 1985. A comparison between implicit and hybrid methods for the calculation of steady and unsteady inlet flows. AIAA Paper No. 851125. $17 \mathrm{p}$.

11. Vlasenko, V. V., E. V. Kazhan, E. S. Matyash, S. V. Mikhaylov, and A. I. Troshin. 2015. Chislennaya realizatsiya neyavnoy skhemy i razlichnykh modeley turbulentnosti $\mathrm{v}$ raschetnom module ZEUS [Numerical realization of implicit scheme and of different turbulence models in ZEUS computing module]. Trudy TsAGI [TsAGI Transactions] 2735:5-49.

12. Vlasenko, V.V. 2015. SOLVER3: dvadtsatiletniy opyt razvitiya i ispol'zovaniya nauchnoy programmy dlya modelirovaniya dvumernykh techeniy s goreniem [SOLVER3: Twenty-year experience of development and usage of scientific code for simulation of two-dimensional flows with combustion]. Trudy TsAGI [TsAGI Transactions] 2735:156-219.

13. Bartlmä, F. 1975. Gasdynamik der Verbrennung. Springer-Verlag. 256 p.

14. Vlasenko, V. V., and A. A. Shiryaeva. 2012. Numerical simulation of non-stationary propagation of combustion along a duct with supersonic flow of a viscid gas. P. I. Mech. Eng. J. Aer. 227(3):480-492.

15. Langener, T., J. Steelant, P. Roncioni, P. Natale, and M. Marini. 2012. Preliminary performance analysis of the LAPCAT-MR2 by means of nose-to-tail computations. AIAA Paper No. 2012-5872. 16 p.

16. Langener, T., J. Steelant, S. Karl, and K. Hannemann. 2013. Layout and design verification of a small scale scramjet combustion chamber. 21st Symposium (Internatial) on Airbreathing Engines Proceedings. Busan, Korea. ISABE Paper No. 20131655. 9-13. 
17. Hannemann, K., J. Martinez-Schramm, S. Laurence, S. Karl, T. Langener, and J. Steelant. 2014. Experimental and numerical analysis of the small scale LAPCAT II scramjet flow path in high enthalpy shock tunnel conditions. Space Propulsion. Cologne, Germany. SP2014-2969350. 8 p.

18. Langener, T., J. Steelant, S. Karl, and K. Hannemann. 2014. Numerical validation of a free-flying scramjet powered vehicle at realistic wind tunnel conditions. Space Propulsion. Cologne, Germany. SP2014-2971766.

19. Shiryaeva, A. A. 2015. Osobennosti chislennogo metoda i rezul'taty testirovaniya programmy ZEUS-S3pp dlya modelirovaniya trekhmernykh techeniy s goreniem [Special features of numerical method and results of testing ZEUS-S3pp code for for simulation of three-dimensional flows with combustion]. Trudy TsAGI [TsAGI Transactions] 2735:220-246.

20. Vlasenko, V.V. 2014. About different ways to determine the heat effect and the combustion efficiency in a flow of reacting gas. TsAGI Science J. 45(1):35-59. 